\title{
Measurement of Cobalt-60 and Cesium-137 Gamma Rays with a Free-Air Chamber
}

\author{
H. O. Wyckoff
}

(October 27, 1959)

\begin{abstract}
Design data for free-air chambers measuring cobalt-60 and cesium-137 gamma rays in roentgens are presented. It has been shown that the Jaffé-Zanstra method of obtaining the saturation current is adequate for air pressures of about 4 to 12 atmospheres. Also, radiation measurements of the gamma rays from cobalt-60 and cesium-137 made by a cavity chamber and a free-air chamber agree to within the experimental errors.
\end{abstract}

\section{Introduction}

The standard instrument for measurement of 5 to $500 \mathrm{kv}$ X-rays in roentgens is the free-air chamber [1]. ${ }^{1}$ Both free-air chambers [2, 3, 4] and cavity chambers are used for measurement in roentgens of the gamma rays from radium, cobalt-60, and cesium-137. Kaye and Binks [2] used both methods to measure the same source of radium and obtained agreement to the order of 1 percent between the two results. However, Taylor and Singer [3] have since pointed out that the free-air chamber measurements by Kaye and Binks were too low by the order of 2 percent because of inadequate plate separation. Ionization from scattered photons, for which no correction was made, may have compensated for part of this error. This contribution was not measured either by Kaye and Binks or by Taylor and Singer. On the other hand Attix and Ritz [5] have indicated that the cavity-chamber measurements of Kaye and Binks require a 1/1/2 percent correction for chamber wall attenuation. An additional possible source of error, inadequate guarding of the collector region, has been pointed out [6] for free-air chamber measurement of low-energy X-rays. Preliminary estimates of this effect on the radium [3], cobalt60 [4], and cesium-137 [4] measurements indicate that the dose rates given there may be up to 5 percent low. Thus, there is not yet an unambiguous comparison of the two methods.

At the present time, neither type of instrument is designated [1] as the standard instrument in the million-volt region. The ultimate selection of one, largely hinges on their individual capability of absolute accuracy. Therefore, measurement of the exposure dose rate of the same radiation sources by the two methods and an analysis of the factors affecting the accuracy seems desirable. Cobalt-60 and cesium-137 gamma-ray sources have already been measured in this laboratory by cavity-chamber

${ }_{1}$ Figures in brackets indicate the literature references at the end of this paper.

methods and an analysis of the accuracy has been performed [5]. Similar treatment is needed for the free-air chamber method and this paper describes such an analysis.

One of the factors which may limit the accuracy of the free-air chamber measurements is the determination of the saturation current. In order to limit the dimensions of the free-air chamber, the ionized air is often at high pressure. Under this condition it is impossible to collect all of the ionization; one must rely on a theoretical extrapolation to an infinite value of the collecting field.

One method of performing this extrapolation, the Jaffé-Zanstra theory [7], has been checked and verified experimentally by Taylor et al. [8] for air pressures of up to $10 \mathrm{~atm} .{ }^{2}$ According to this theory [7], a plot of the inverse of the collected ionization current versus a particular function of $x$, $f(x)$, should give a straight line and the intercept at $f(x)=0$, where the field strength is infinite, should give the inverse of the saturation current. Here $x=c(V / D P)^{2}$; where $V=$ collecting potential in volts ; $D=$ plate seperation in $\mathrm{cm} ; P=$ pressure in atmospheres; and $c$ is empirically determined from the requirement that theinverse of the collected ionization versus $f(x)$ should be a straight line. Curves of $f(x)$ versus $x$ are available in the literature [7]. However, at least two very different values of $c$ have been published $[8,9]$. The latter reference also recommends a field strength per atmosphere of at least $80 \mathrm{v} \mathrm{cm}^{-1}$ $\mathrm{atm}^{-1}$, contrary to the earlier work [8] where fields of less than $10 \mathrm{v} \mathrm{cm}^{-1} \mathrm{~atm}^{-1}$ give data that fall on the straight line plot.

A second method of extrapolation to an infinite field has been suggested by Kara-Michaelova and Lea [10]. They plot the inverse of the measured ionization current versus the inverse of the collecting potential. For sufficiently high potential, a linear relation exists which may be extrapolated to an

2 The authors neglected the correction for the scattered photon contribution. When such a correction is made for cesium-137 gamma rays, the extrapolated When such a correction is made for cesium-137 gamma rays, the extrapolated
current per atmosphere at $10 \mathrm{~atm}$ is at least 2 percent less than that at $1 \mathrm{~atm}$. current per atmosphere at 10 atm is at least 2 percent less than that at 1 atm.
The correction should be larger for the $300-\mathrm{kV} \mathrm{X}$-rays used for this test by Taylor 
infinite collection field. The authors also pointed out the equivalence of the two methods for obtaining saturation current when the collecting potentials are sufficiently high. Therefore, an exploration of the feasibility of the suggested extrapolation methods seems desirable for the present application. At the same time, it is worthwhile to obtain the radial distribution of the ionization from the primary electrons and from the scattered photons and the distance required for electron equilibrium. These are necessary [11] design data for the construction of a freeair chamber for measuring the gamma rays from cesium-137 and cobalt-60 in roentgens.

The main objective of this report is, therefore, to carefully measure a cobalt-60 and a cesium-137 gamma-ray source with a free-air chamber, and compare these measurements with those obtained with the cavity chamber. With a careful analysis of the accuracy of each type of measurement it may be possible to indicate which is capable of greatest accuracy at the present time and therefore, can be designated as the standard instrument in the millionvolt region. However, the free-air chamber measurements require an exploration of the feasibility of the extrapolation methods for saturation and the determination of the design data necessary for the construction of free-air chambers for measuring cesium137 and cobalt-60 gamma rays in roentgens.

\section{Experimental Arrangement}

A small beam of gamma rays from a radiation source is defined by an accurately known aperture (approximately $2 \mathrm{~cm}$ in diameter) in the shutter- diaphragm (fig. 1). This aperture is at the end of a cylindrical hole parallel to, but displaced from the axis of the cylindrical shutter-diaphragm. Rotation of the shutter cuts off the beam and permits measurement of the leakage radiation without introducing a modification of the scattering conditions. The beam passes first through a thin aluminum window $(0.8 \mathrm{~mm}$ thick), then midway between and parallel to the two vertical plates of a parallel-plate ionization chamber, and then through a glass exit window. The ionization chamber is sealed inside a large tank filled with air under high pressure so that the mass of air between the plates is adequate for totally absorbing all the energy of the highest energy electrons produced by the beam of gamma rays.

One of the plates of the ionization chamber is maintained at a high potential with respect to ground (see sec. $\mathrm{A}-\mathrm{A}$ of fig. 1 ); the other is divided into 3 collecting regions (plates marked 2, 4, 5a, 5b, 6a, 6b, $7 \mathrm{a}, 7 \mathrm{~b}$, and 9 ) and 4 large and 6 small grounded guard plates (see elevation view of fig. 1). The center collecting region is divided into 7 separate insulated plates (marked 4, 5a, 5b, 6a, 6b, 7a, and 7b). Each collector plate is separated from the adjacent plate by an air gap of $0.5 \mathrm{~mm}$. A flat grounded plate is installed between the collector plates and the tank at about $3 \mathrm{~cm}$ from the collector plate to reduce the possibility of ionization collection from behind the collector plate.

The ionization to any of the collecting plates can be measured. During an exposure the plates which are not used for ionization measurement are grounded. After careful alinement of the plate

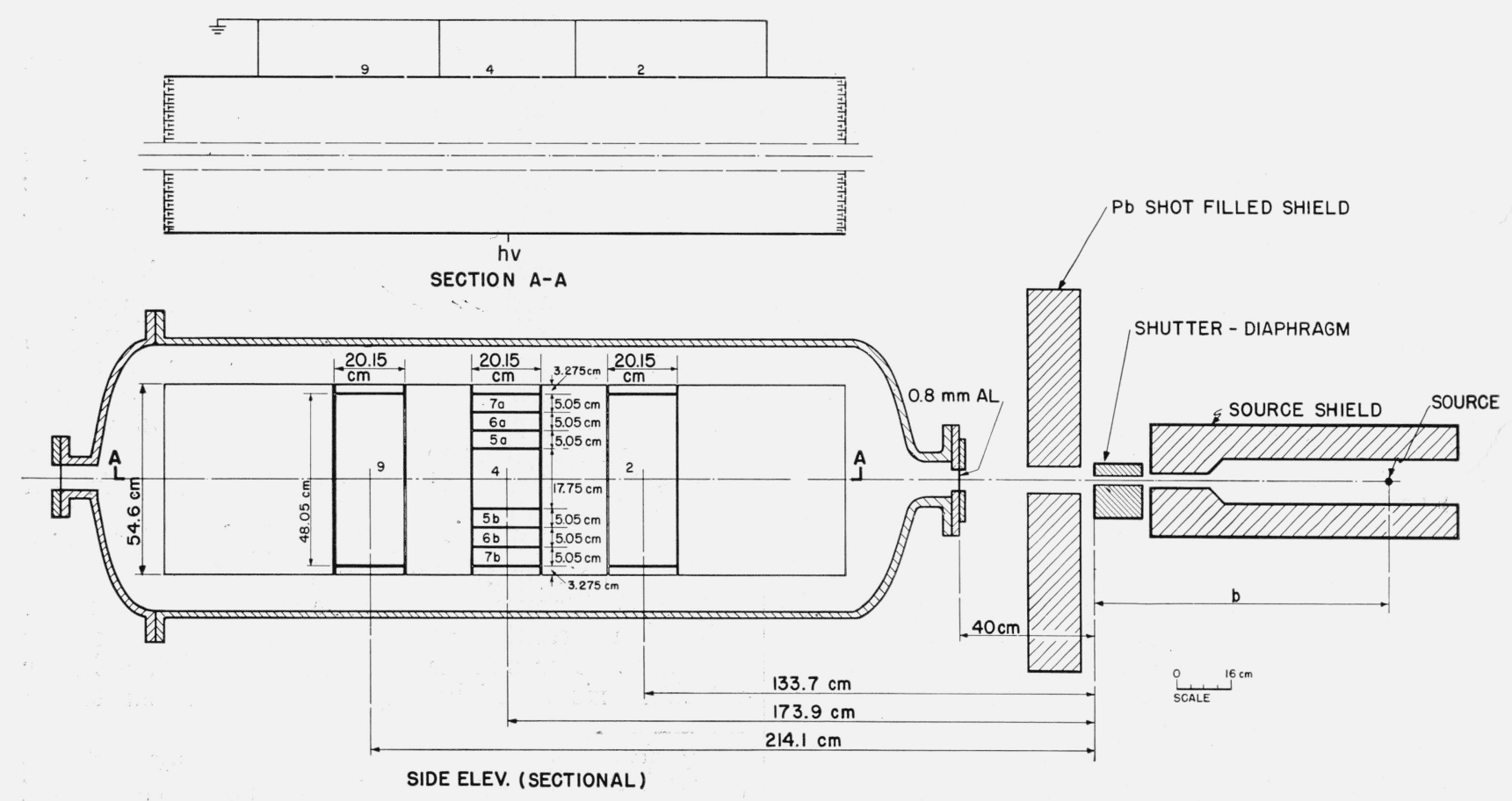

Figure 1. The experimental arrangement. 
system, the displacement of the plane of each collector from the adjacent guard plate is measured. Generally, this is less than $0.03 \mathrm{~mm}$ but increases to as much as $0.1 \mathrm{~mm}$ very near the top of plate 2 and the bottom of plate 9 because of warpage of these plates. This causes a distortion of the field. However, previous measurements [11] indicate that as the ionization collected in this latter region is small, this distortion should cause no more than 0.1 percent error in the collected ionization.

This collector plate arrangement provides a means for determining the adequacy of the plate separation at a given pressure and also provides a means for determining the air absorption of the gamma-ray beam. With proper guarding of the electric field, each plate collects ionization from a volume defined by the plate height, the length of $20.15 \mathrm{~cm}$ (fig. 1), and the distance between the collector and highvoltage plates. Thus, by observing the relative amount of ionization collected by each of the plates 4 to 7 , it is possible to determine if the effective plate separation (the product of plate separation and pressure) is adequate to collect all the ionization from electrons produced in the beam.

The ionization current collected by plate 9 is smaller than that collected by plate 2 , due to air absorption. Therefore, the ratio of these two measurements may be used to compute the air absorption coefficient. Comparison of these computed values at the different pressures for each source provides an independent estimate of the precision of the current measurements. A comparison with the theoretical absorption coefficient indicates whether there is a difference in any systematic error between the current measurements to the two plates.

Guard bars are used to assure that the grounded tank does not distort the field in the collecting region. These are $T$ cross section aluminum bars equally spaced between the chamber plates and parallel to these. In order to avoid extra photon attenuation and scattering, vertical bars are not used in either the front or back of the guard system where they might be struck by the beam (fig. 2). In these regions, fine aluminum wires are used instead of bars. A resistance network with a tap for each bar divides the potential uniformly between the high voltage and ground. A determination of the ability of these guard bars to eliminate the effect of the pressure tank on the electric field between a collector and the high-voltage plate requires an auxiliary experiment. As pointed out previously [5, 11], this is accomplished by noting the variation in collected ionization current as the potential of the tank is varied. Ionization measurements to each of the collectors indicate no variation greater than \pm 0.1 percent as the tank potential varies from ground potential up to the potential of the high-voltage plate. Thus, the tank produces no distortion of the collecting field which changes the collecting volume by more than the precision of measurement (about \pm 0.1 percent). All subsequent measurements are performed with the tank at ground potential.

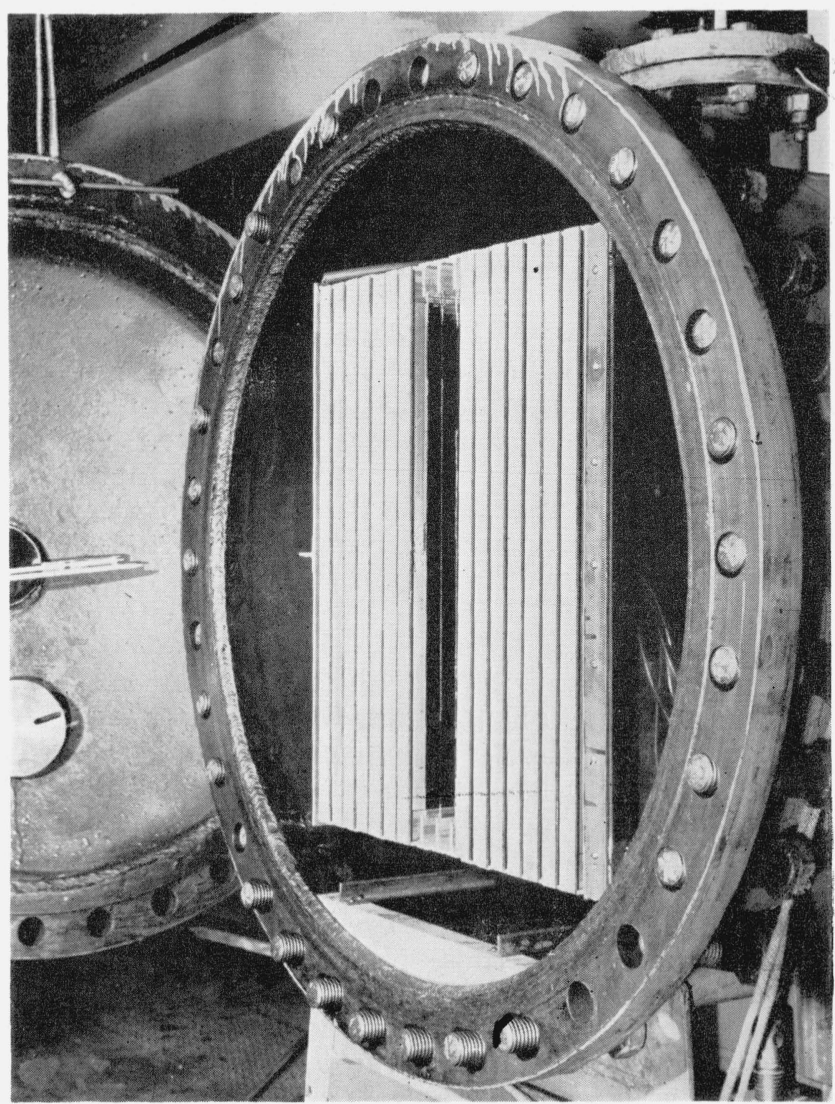

Figure 2. A view of the back of the tank with the end bell removed showing the guard bars for the chamber.

The air pressure in the tank is determined from the reading of a bourdon-type gage and the barometric pressure. The gage is calibrated by a piston gage and shows a short-time (over about 2 yr) maximum driit of calibration of about \pm 0.2 psi and a longtime stability (over $8 \mathrm{yr}$ ) of about \pm 0.3 psi except for a progressive zero shift $(0.4$ psi over $8 \mathrm{yr})$. For pressures of less than $5 \mathrm{~atm}$, a mercury manometer also is used. In this range, the two methods agree with each other to within 0.2 percent. Filters and an ice-cooled condenser in the pumping system, as well as silica gel on the inside of the tank, maintain a low water vapor pressure. Mass spectrometric tests of the air under pressure indicate that the composition is the same as room air.

Separate thermocouples are used to test for possible temperature differences of the high-pressure air in the various ion collecting regions of the tank, and to calibrate as a function of pressure a mercury thermometer placed inside the tank near the exit window. For this test, thermocouples are placed at the center of each of the collecting regions as well as near the thermometer. After a short stabilizing period, all of the thermocouples give temperatures agreeing to better than $0.2^{\circ} \mathrm{C}$. Moreover, the calibration of the thermometer at a given pressure appears to be constant to $0.2^{\circ} \mathrm{C}$, although its calibration varies by nearly $1^{\circ} \mathrm{C}$ over the pressure range used. 
In order to determine the ionization due to scattered photons, the ionization current also is obtained with a $10-\mathrm{cm}$ diam $2-\mathrm{m}$ long, Bakelite tube surrounding the collimated beam of photons inside of the tank. This tube is of sufficient thickness (about $3 \mathrm{~mm}$ ) so that it stops all electrons originating in the air within the tube, and of sufficient diameter so that it is not struck by the primary beam of gamma rays.

To reduce the possible distortion of the electric field by the tube, colloidal graphite is painted in strips along the length of the tube. Adjacent strips are insulated from each other by uncoated tubing. Each of these strips is maintained at a potential which minimizes the electric field distortion by connecting it to the proper guard bars. Observation of the ionization from background radiation ${ }^{3}$ with and without the tube in place gives an estimate of the field distortion produced by the tube. With the tube in place the current should be the same as those without the tube for plates $5 \mathrm{a}, 5 \mathrm{~b}, 6 \mathrm{a}, 6 \mathrm{~b}, 7 \mathrm{a}$, and $7 \mathrm{~b}$. However, with the tube in place plate 4 will not collect ionization from the area in the tube shadow, that is, between the tube and the high voltage plate and within the tube but the difference can be computed from the geometry. The measured current to the different collectors are in the same ratio to within 5 percent as those predicted from the geometry. As the scattered photon contribution is only 2 or 3 percent of the total ionization, field distortion caused by the presence of the tube does not affect the final result. The ionization produced by electrons generated by the primary photons is, within the precision of measurement, proportional to the difference between the ionization measured with ${ }^{4}$ and without this tube.

The ionization-current measuring system consists of a null indicating electrometer with feedback, a carefully calibrated condenser, and a variable bucking potential provided by a potentiometer. Timing of each exposure is accomplished by means of a scaler and a 1-kc oscillator [12]. This chronograph gives timing accuracies of about 0.01 percent for the times used here.

Measurements are made with the shutter closed in order to correct for the background. As the shutter-closed measurement is not changed when a solid lead plug is inserted into the hole in the leadshot-filled shield, the leakage radiation through the shutter is negligible and the shutter-closed measurement indicates the background.

In addition to corrections for leakage radiation and air-scattered photons, it is also necessary to correct for any ionization from photons scattered by the source shield or diaphragm. Consider any rays which are scattered by the source shield and then pass through the diaphragm aperture. It may be shown for the geometry of figure 1 that the path of

\footnotetext{
3 The background radiation is assumed to produce a uniform density of ionization over the whole collecting region.

${ }_{4}$ A slignt correction is required for the shadow cast by the tube and a larger correction for lack of saturation. The reduction of the scattered photon ionization by photon absorption in the tubewall is much smaller than 0.1 percent of the tubeout ionization. Therefore this reduction can be neglected.
}

such rays will pass through the collector regions at distances greater than $5 \mathrm{~cm}$ from the axis of the primary beam. Thus, the ionization that they produce in the collecting region will be measured both with and without the Bakelite tubing in place, and the difference in readings will not include ionization either from photons scattered by the source shield or from the air in the chamber.

The effect of scattering from the diaphragm is explored by comparing the ionization current with diaphragms $15 \mathrm{~cm}$ and $22.5 \mathrm{~cm}$ long.

The source holders are made as light as is consistent with dimensional stability so as to reduce the amount of scattered radiation from them. These source holders fit into the large hole in the rear end of the source shield. The position of the source is determined by observing the position of the end of the long rod attached to each source holder. The radiation is measured perpendicular to the axis of the $3.2 \mathrm{~mm}$ diam by $3.2 \mathrm{~mm}$ long cobalt-60 source and along the axis of the $4.6 \mathrm{~mm}$ diam by $5 \mathrm{~mm}$ long cesium-137 source. The cobalt-60 source is encapsulated in aluminum with a $1.6 \mathrm{~mm}$ thick wall and the cesium-137 in $1 \mathrm{~mm}$ thick stainless steel.

A separate piece of equipment similar to that previously used [13] provides a means of determining the distance required for electronic equilibrium [11]. A grid chamber (fig. 3), made of colloidal-graphite-

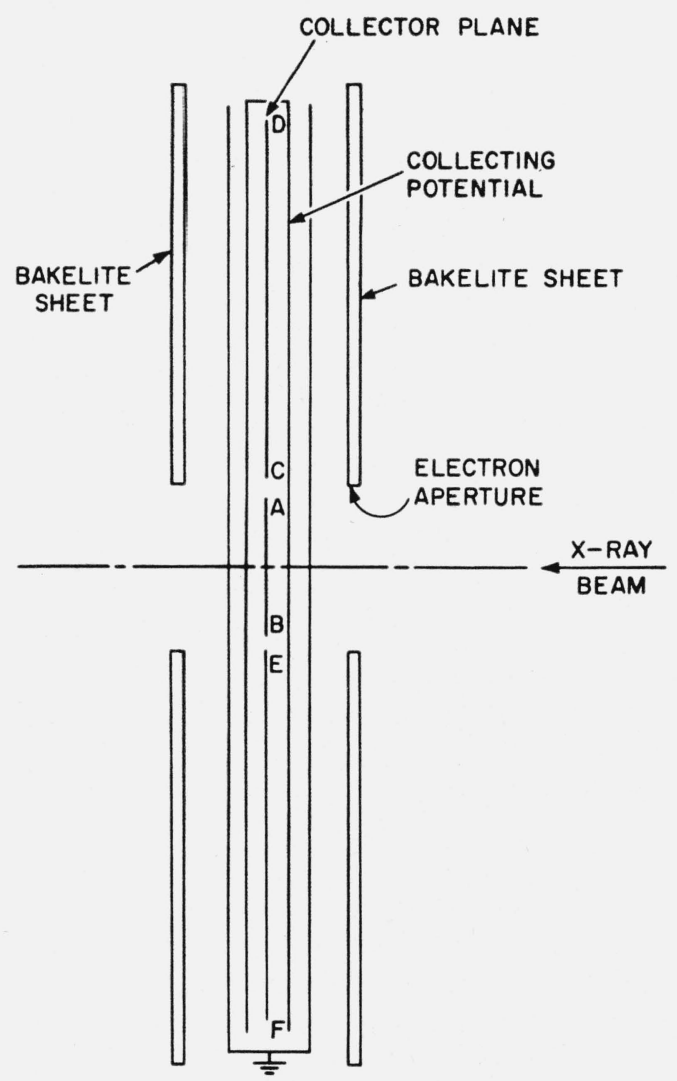

Figure 3. Schematic plan view of the thread chamber and electron diaphragms.

For simplicity the threads in each plane are drawn as a single line. 
coated nylon threads $(\sim 0.2 \mathrm{~mm}$ diam $)$ in 5 parallel planes spaced about $1 \mathrm{~cm}$ apart, is placed in the pressure tank with the planes perpendicular to the gamma-ray beam. The threads in any one plane also are spaced about $1 \mathrm{~cm}$ apart. The threads in the central plane form a collector at near ground potential, and the two adjacent planes are connected to the collecting potential. The collector plane has two collecting regions separated by uncoated thread for insulation. A central collecting region, which is coaxial with the gamma-ray beam, is about $6.5 \mathrm{~cm}$ diam (AB in fig. 3). The outer collecting region has an inner diameter of $8.5 \mathrm{~cm}(\mathrm{CE})$ and an outer diameter of $51 \mathrm{~cm}$ (DF). The gamma-ray beam diameter at the position of the collector plane is about $6.5 \mathrm{~cm}$. Thus, the central collector measures the ionization in a cylindrical region of about $2 \mathrm{~cm}$ depth and a diameter of $7.5 \mathrm{~cm}$, and the outer collector measures the ionization in a ring of about $2 \mathrm{~cm}$ depth, $7.5 \mathrm{~cm}$ inner diameter and about $51 \mathrm{~cm}$ outer diameter.

A circular electrically-conducting Bakelite sheet (electron diaphragm), which is thicker than the range of the electrons produced by the gamma rays in air, is positioned perpendicular to the gamma-ray beam and in front of the grid chamber. A similar sheet is positioned behind the chamber. Each sheet has a center hole, which is slightly larger than the gamma-ray beam and coaxial with it, and each can be moved along the beam by remote control from outside the pressure tank.

\section{Results and Discussion}

The ionization current to each of the collector plates is measured both with and without the Bakelite tube and for a variety of pressures and collecting potentials. Data are obtained for five different pressures from 3.96 to 10.75 atm with the gamma rays from cesium-137 and for pressures of 10.75 and 12.27 atm for cobalt-60 gamma rays in order to choose a value for the Jaffé-Zanstra constant, $c$. With the Bakelite tube in place part of the ionization from the scattered photons is produced in the shadow of the tube and is thus not collected on plates 2,4 , and 9. However, the ionization produced in a ring about the axis of the beam decreases only slowly with increase of the ring radius ${ }^{5}$. Therefore, it is relatively simple [14] to make a correction for this shadowing, and also to change from the rectangular coordinate system of measurement for plates 4 to 7 to a cylindrical set of coordinates. For a given pressure and collecting potential, the difference between the ionization measured without the tube in place and the shadow-corrected determination with the tube in place is proportional to the ionization produced in the collecting length $(20.15 \mathrm{~cm})$ by the electrons generated by the primary gamma rays.

\footnotetext{
${ }^{5}$ Scattering in the source shield or collimator might distort this monotonic decrease but such effects prove to be small in the present arrangement. Correction is still required for lack of saturation.
}

If the pressure is too low, so that some of the energy of the electrons is dissipated in the plates, a linear extrapolation to larger radii may be performed [14] on a log-current-loss versus radius plot. For the data reported here, this extrapolation does not exceed $2 / 3$ percent.

Data from the large range of pressures used with cesium-137 gamma rays permits a study of the techniques for extrapolating to infinite collecting fields. Figure 4 shows plots of the inverse of the ionization currents, ${ }^{6} \quad I$, against the Jaffé-Zanstra parameter, $f(x)$. Four values of $c$ are used to obtain $f(x)$ in order to determine the value of $c$ which gives a straight line. The value of $1.24 \times 10^{-4}$ is that used by Taylor et al., [3] and by Grove [4]. It is seen that the point for the largest value of $f(x)$ falls well below the straight line through the remaining points. However, for both $c=3.2 \times 10^{-5}$ and $c=2.5 \times 10^{-5}$, all data fall on straight lines with slightly different intercepts at $f(x)=0$ (infinite collecting field). For $c=10^{-5}$, the points for large values of $f(x)$ fall above the straight line for the remaining points. The proper intercept on the $1 / I$ axis therefore probably lies between that obtained for $c=10^{-5}$ and for $c=1.24 \times 10^{-4}$.

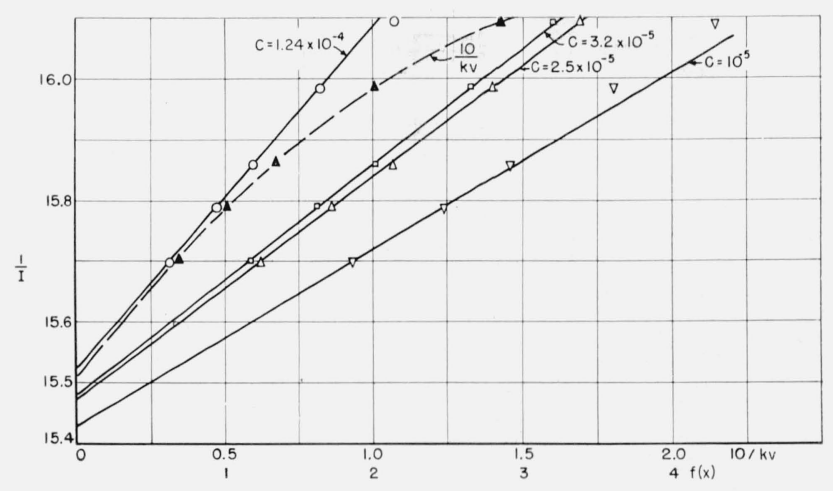

Figure 4. Sample plots of the extrapolation procedure with cesium-137 gamma rays at a tank pressure of $3.96 \mathrm{~atm}$.

Four Jaffé-Zanstra type plots are indicated using different values of $c$. A Kara-Michaelova and Lea type plot (marked $10 / \mathrm{kV}$ ) does not give a straight line relation.

Also included in figure 4 is a plot (marked $10 / \mathrm{kv}$ ) of the inverse of the ionization current, $I$, against the inverse of the collecting potential (Kara-Michaelova and Lea). The intercept on the $1 / I$ axis for the inverse collecting potential plot is indeterminate as the points do not fall on a straight line even for the three smallest values of $1 / I$, where the effective electric field strengths are about 171, 114, and $85.5 \mathrm{v} \mathrm{cm}^{-1} \mathrm{~atm}^{-1}$.

While the uncertainty in the intercept by these two methods is small at this pressure because the extrapolation is only about $1 \frac{1}{2}$ percent, the uncertainty becomes larger at the higher pressures where the extrapolation is as large as 10 percent. Fortunately, there is another criterion for choosing the proper extrapolation procedure.

\footnotetext{
${ }_{6}^{6}$ Tube-in minus tube-out determination after correction for tube shadowing.
} 
The exposure dose rate at the aluminum window is independent of the pressure in the tank. The exposure dose rate is proportional to the ionization current per atmosphere extrapolated to an infinite field and corrected for air attenuation, i.e., to $I_{\infty} e^{P d \mu} / P$. Here $I_{\infty}$ is the current at infinite field, $P$ is the pressure in atmospheres, $d$ is the distance between the center of the collector plate and the entrance window, and $\mu$ is the linear absorption coefficient of the gamma rays in air. Values of $I_{\infty} e^{P d \mu} / P$ at different pressures are listed in table 1 for cesium137 gamma rays. These results verify the earlier conclusion that a proper extrapolation is obtained for a value of $c$ between $1.24 \times 10^{-4}$ and $10^{-5}$. As the extrapolated values for $c=2.5 \times 10^{-5}$ show a slightly smaller deviation from the average than $c=3.2 \times 10^{-5}$, this value is used for all subsequent determinations for both the cesium-137 and the cobalt-60 source.

TABLE 1. $1011 \frac{I_{\infty}}{P} e^{P d \mu}$ for cesium-137

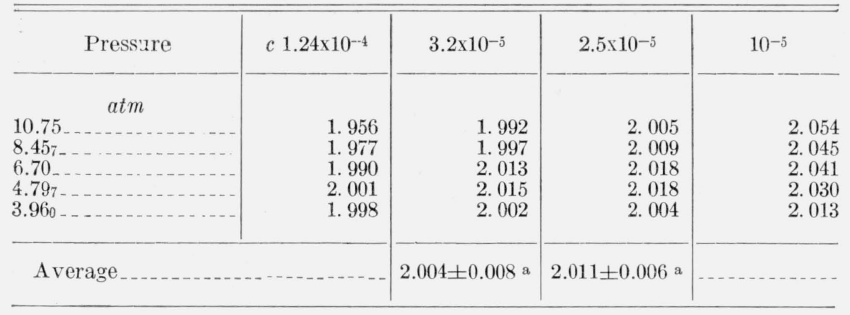

a Mean deviation.

The computations of $e^{P d \mu}$ assume that the photons which generate the electrons are attenuated for a distance, $d$, in air. Actually, most of the electrons which produce the ionization in the collecting region are generated before and a few after the photons reach the collecting region. The amount by which $d$ must be reduced because of this is the distance, $a$, from the center of the collecting region to the mean position of origin of the electrons producing ionizations in the collecting region. As the value of $a$ is nearly a constant fraction of the distance for electronic equilibrium for the photon energies considered here, it is convenient to first determine the distance $^{7}$ for electronic equilibrium. These distances for the two sources may be obtained from the thread chamber data.

Results obtained with the thread chamber for cesium-137 gamma rays at $6.7 \mathrm{~atm}$ are shown in figure 5 and for cobalt-60 gamma rays at 12 atm in figure 6 . The solid curves give the ionization current to the outer collector ring for different positions of each Bakelite sheet with the other sheet positioned as close as possible to the collecting region; the dotted curves are for the ionization current to the central collector. Initially the solid curves rise rapidly with the distance between the sheet and the collecting region, but eventually reach a plateau. The rise indicates that electrons generated at larger distances from the collecting plane are

7 This distance is also ne zessary for free air chamber desig $n$.

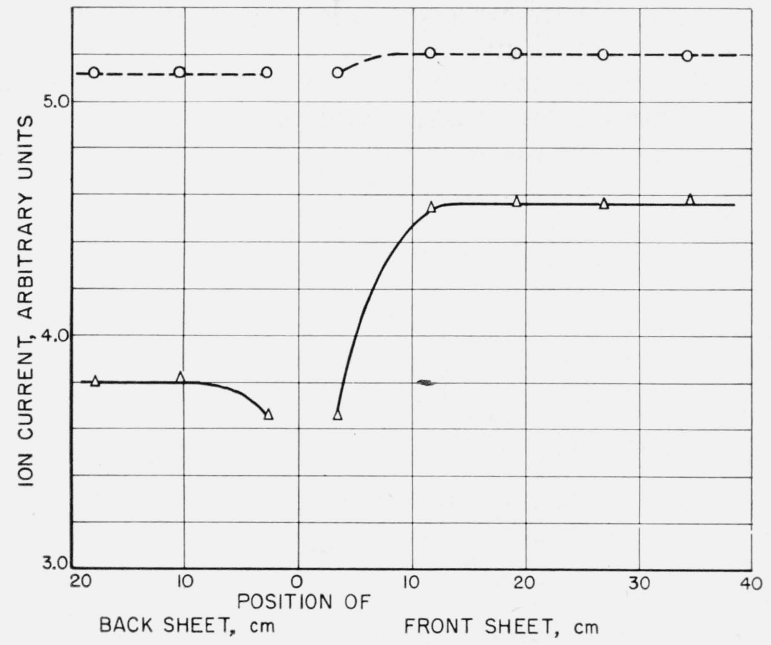

FiguRE 5. Data obtained from the thread chamber and cesium187 gamma rays at $6.7 \mathrm{~atm}$.

1. The solid curves give the ionization current to the outer collecting ring and the dotted curve"to the center electrode.

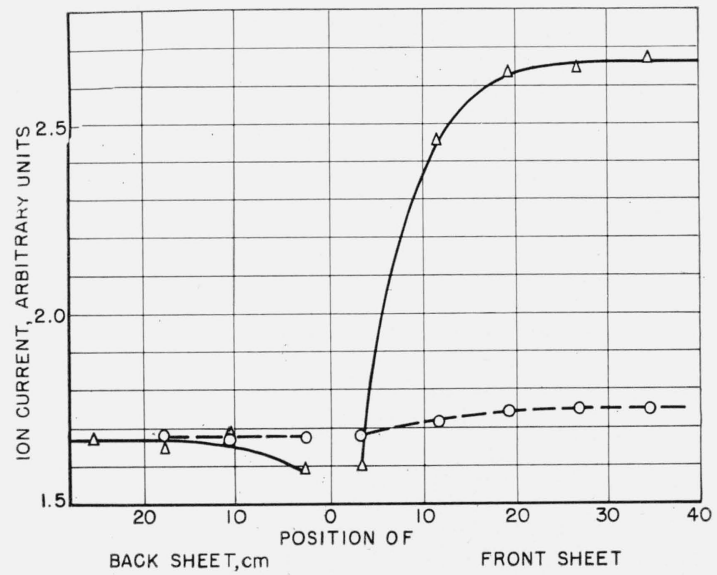

FIGURE 6. Data obtained from the thread chamber and cobalt-60 gamma rays at $12 \mathrm{~atm}$.

The solid curves give the ionization current to the outer collecting ring and the dotted curve to the center electrode.

contributing to the ionization. The distance at which the ionization becomes constant is the distance for electronic equilibrium. From the solid curves it appears that the distance required for electronic equilibrium with cobalt-60 gamma rays is about 350 cm-atm in front of the collector and about 180 $\mathrm{cm}$-atm behind the collector. The corresponding distances for cesium-137 are 90 and $50 \mathrm{~cm}$-atm. Aglintsev and Ostromukhova [15] also obtained a distance of about $350 \mathrm{~cm}$-atm for cobalt- 60 gamma rays.

The mean distance from the collecting region to the mean position of origin of electrons producing ionization in the collecting region is, of course, less than the equilibrium distances. Calculations by Roesch [16] indicate the distance from the collecting region to the mean position of origin of the electrons is about 0.3 times the distance for electronic equilibrium. Aglintsev and Ostromukhova [15] computed 
values of 0.36 for cobalt-60 and 0.34 for cesium-137 gamma rays. For these distances in a free-air chamber they compute the transmission, $K=e^{-P a_{\mu}}$, of cesium-137 and cobalt-60 gamma rays to be 0.996 and 0.992 , respectively. In a carbon-walled cavity chamber, because of the lower effective absorption coefficient there, these transmissions are 0.998 and 0.995. These factors must be multiplied by $e^{P d \mu}$ in order to obtain the attenuation of the photons whose electrons produce the measured ionization.

The attenuation cofficients for the gamma-ray beams are computed from data obtained for plates 2 and 9 . The difference in the currents obtained with the tube out and with the tube in, after correction for the shadowing and saturation, gives the current produced by the primary beam. The ratio of the plate-2-to-plate- 9 currents are given in table 2 for different pressures with the two sources. The linear absorption coefficient computed from the ratio and that given by Grodstein [17] are also shown. The maximum deviation of the computed absorption coefficient from the mean is approximately 6 percent and the maximum difference between the experimental and theoretical values is also 6 percent. The first number indicates that the precision of the current ratio is at least 0.5 percent and the second that the systematic errors if they exist for two current determinations are equal within the precision of measurement. As there is no reason to assign different precisions to the two current determinations, the precision of each is about 0.35 percent.

TABLE 2. Air absorption coefficient

\begin{tabular}{|c|c|c|c|c|}
\hline \multirow{2}{*}{ Source } & \multirow{2}{*}{ Pressure } & \multirow{2}{*}{$\frac{I_{2}}{I_{9}}$} & \multicolumn{2}{|c|}{$\mu \mathrm{cm}^{-1}$} \\
\hline & & & Experiment & Grodstein [17] \\
\hline $\begin{array}{l}\mathrm{Cs}^{-137}- \\
\mathrm{Co}^{-60}+\end{array}$ & $\begin{array}{l}\text { atm } \\
10.75 \\
8.457 \\
6.70 \\
4.797 \\
12.27 \\
10.75\end{array}$ & $\begin{array}{l}1.094 \\
1.068 \\
1.057 \\
1.042 \\
1.076 \\
1.069\end{array}$ & $\begin{array}{r}1.04 \times 10^{-4} \\
0.96 \times 10^{-4} \\
1.04 \times 10^{-4} \\
1.06 \times 10^{-4} \\
7.5 \times 10^{-5} \\
7.7 \times 10^{-5}\end{array}$ & $1.0 \times 10^{-4}$ \\
\hline
\end{tabular}

Possible scattering from the diaphragm is investigated by obtaining data with both a $15 \mathrm{~cm}$ and a $22 \frac{1}{2} \mathrm{~cm}$ long diaphragm. The radiation scattered through the diaphragm and into the chamber from the source holder is decreased slightly by lengthening the diaphragm. However, this decrease is estimated to be less than 0.1 percent of the electron contribution to the ionization. Such a small decrease is not observable from the tube-out measurement as this is less than the precision. Large angle scattering from the shutter-diaphragm is considerably attenuated by the steel surrounding the entrance window of the pressure tank. Probably for this reason no difference is obtained in the current to plate $7 \mathrm{a}$ and $7 \mathrm{~b}$ when the diaphragm length is increased. Currents to plates $5 \mathrm{a}, 5 \mathrm{~b}, 6 \mathrm{a}$, and $6 \mathrm{~b}$ are decreased slightly $(0.1 \%$ of the electron contribution) as the diaphragm length is increased for the tube-out measurement. There is a similar decrease for the tube-in measurement. Therefore, the differences in the tube-out and tube-in measurements are independent of diaphragm scattering.

From the measured currents to plates 4, 5, 6, and 7 , it is possible [14] to compute the loss in ionization (electron loss) obtained beyond different radii and also the contribution to the ionization provided by the scattered photons. In order to compare the results obtained at different pressures for each source, the radii at each pressure are corrected for the diameter of the gamma-ray beam [13] and for the density of the air. The abscissa of figures 7 and 8 is in equivalent centimeters at 1 atm for a zero diameter beam. It is seen that the data of figure 7 agree to within about \pm 0.2 percent for different pressures and that the data of figure 8 agree to within about \pm 0.1 percent for different pressures.

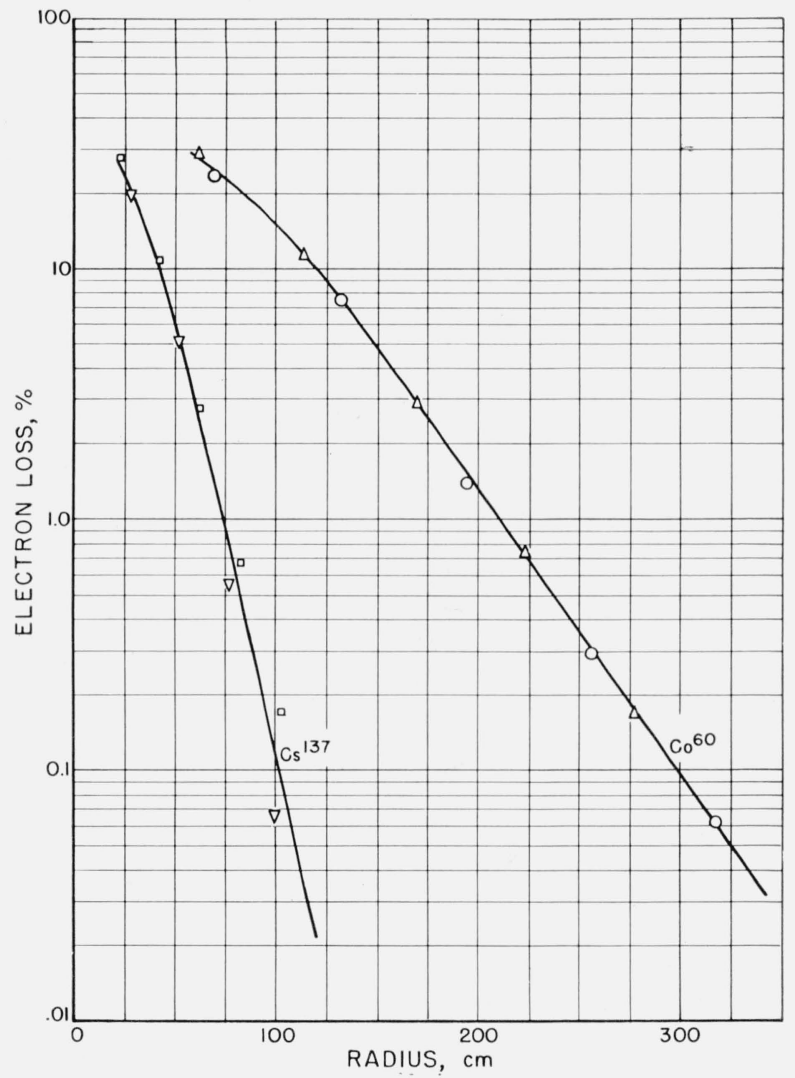

Figure 7. The loss of ionization from primary photons beyond different radii for cobalt-60 and cesium-137 gamma rays.

Results have been corrected to $1 \mathrm{~atm}$ and for the beam diameter. Results with cesium-137 at $3.96 \mathrm{~atm}=\odot$, at $4.797 \mathrm{~atm}=\nabla$; with cobalt- 60 at $12.27 \mathrm{~atm}=\odot$, at $10.75 \mathrm{~atm}=\triangle$.

The gamma ray output, $G$, in roentgens per hour at $1 \mathrm{~m}(\mathrm{rhm})$ for each source is obtained by substituting numerical values in eq (1).

$$
G=\frac{(3,600)(2.998)\left(10^{9}\right) I_{\infty} b^{2} K e^{P d_{\mu}} e^{0.08 \mu} \mathrm{Al} e^{100(b+0 \cdot 40) \rho \mu / 0.001293}}{P A D L} \mathrm{rhm}
$$




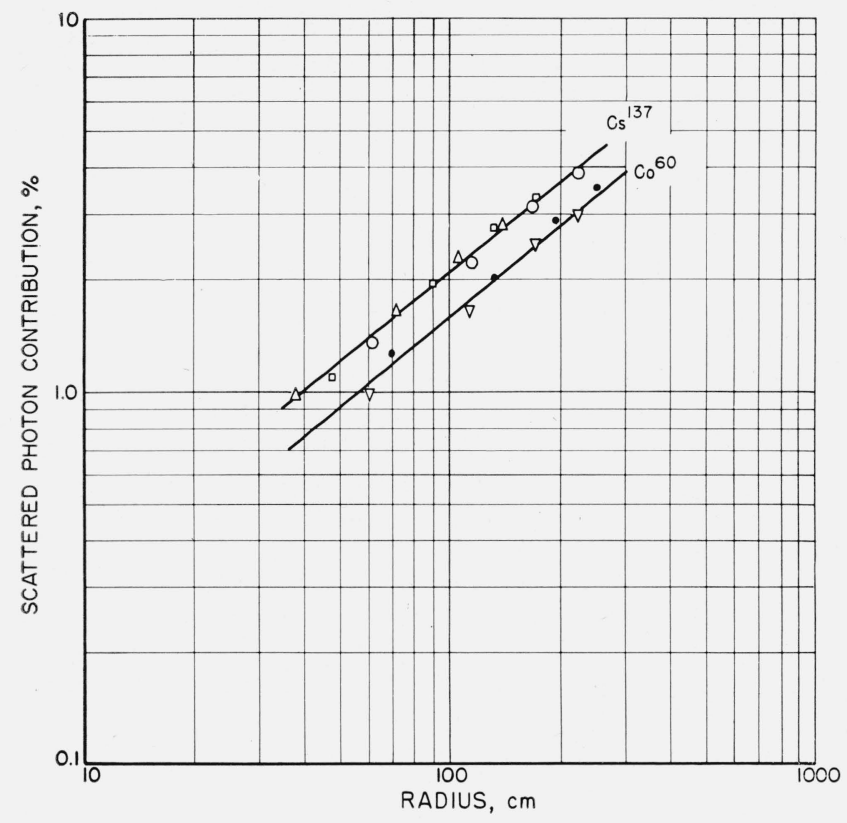

Figure 8. Contribution of secondary photon ionization in percent of total primary electron ionization for different radii from the cesium-137 and cobalt-60 beam.

Results have been corrected to $1 \mathrm{~atm}$ and for the beam diameter. Results with cesium- 137 at $10.75 \mathrm{~atm}=\odot$, at $8.457 \mathrm{~atm}=\circlearrowright$, at $6.70 \mathrm{~atm}=\triangle$; with cobalt -60 at $12.27 \mathrm{~atm}=\odot$, at $10.75 \mathrm{~atm}=\nabla$.

where

$$
3,600=\sec \mathrm{hr}^{-1}
$$

(2.998) $\left(10^{9}\right)=$ esu of charge coulomb ${ }^{-1}$,

$I_{\infty}=$ ionization current in amperes produced by the primary beam in the collecting region,

$b=0.871 m$ (see fig. 1),

$K=0.992$ for cobalt-60 and 0.996 for cesium-137,

$P=$ air pressure in atmospheres,

$d=$ distance from collecting region to entrance window, $133.9 \mathrm{~cm}$,

$\mu=$ air absorption coefficient in $\mathrm{cm}^{-1}$ $\operatorname{atm}^{-1}$

$\mu_{A l}=$ aluminum absorption coefficient in $\mathrm{cm}^{-1}$

$e^{100(b+0.40) \rho \mu / 0.001293}=$ air absorption correction between source and window,

$\rho=$ density of laboratory air in $\mathrm{g} \mathrm{cm}^{-3}$,

$0.001293=$ density of air at a pressure of 760 $\mathrm{mm}$ mercury and a temperature of $0^{\circ} \mathrm{C}$ in $\mathrm{g} \mathrm{cm}^{-3}$,

$A=$ measured area of aperture of diaphragm, $3.147_{8} \mathrm{~cm}$,

$D=\frac{\text { effective area of aperture }}{A}$, and

$L=$ collector length $(20.15 \mathrm{~cm})$.

The effective area of the aperture is greater than the area $A$ because of photon leakage through the edges of the aperture [3]. The computed factor, $D$, allows for this leakage.
Table 3 gives the rhm for each of the sources computed from the equation with the data obtained with the pressure chamber.

TABLE 3. Output determinations

\begin{tabular}{|c|c|c|c|}
\hline Source & Free-air chamber & Cavity chamber & Difference \\
\hline $\mathrm{Co}^{-60} \ldots$ & $0.756 \mathrm{rhm}$ & 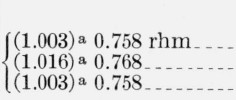 & $\begin{array}{ll}\% & \\
& 0.3 \\
1.6 \\
0.3\end{array}$ \\
\hline $\mathrm{Cs}^{-137} \ldots$ & $2.61_{1} \ldots \ldots \ldots \ldots$ & $\left\{\begin{array}{l}(1.007) \text { a } 2.569-\ldots \\
(1.026) \text { a } 2.619-\ldots \\
(1.013) \text { a } 2.586 \ldots\end{array}\right.$ & $\begin{array}{l}1.6 \\
0.3 \\
1.0\end{array}$ \\
\hline
\end{tabular}

a The numbers in parentheses are the ratio of the stopping power of carbon to that of air for "Bakker-Segre," "Caldwell," and "NCRP" mean excitation potentials, respectively, for each of the sources.

The carbon cavity chamber previously used by Attix and Ritz [4] is also used to measure the sources. The largest single uncertainty in such a determination is in the stopping power. Attix and Ritz have indicated two sets of values for the mean excitation potential to be used in the stopping power equation. For one set, labeled "Bakker and Segre," they give 76.4 and $80.5 \mathrm{ev}$ for carbon and air respectively; for the other set, labeled "Caldwell," they give 78 and 94.9. An analysis by a task group of the National Committee on Radiation Protection and Measurements [18] of the information presently available provides values of 78.4 and $85 \mathrm{ev}$ for carbon and air respectively. The resulting stopping power ratios (in parentheses) obtained after taking into account the density correction and the outputs determined from the cavity chamber data for each are listed in table 3 .

The emission constant of the cobalt-60 source may also be computed. The activity in curies is obtained from the measured value of Minton [19] with a radioactive decay correction. Corrections are also applied to the values in table 2 for photon absorption in the aluminum capsule (1.011) and in the cobalt-60 cylinder $(1.030)$. The resultant emission constant is $12.7_{3} \mathrm{r} \mathrm{cm}^{2} \mathrm{mc}^{-1} \mathrm{~h}^{-1}$. The theoretical value for this constant is 12.9 if the average energy to produce an ion pair is $34.0 \mathrm{ev}$. In view of the uncertainty in the activity determination $(1 \%)$ and the uncertainty in the decay correction for the four years between the activity and ionization-chamber determinations, the agreement seems reasonable.

\section{Accuracy}

In order to assign an accuracy to the free-air and cavity-chamber values in table 3 , it is necessary to review the accuracy of each of the factors used in determining the values. These factors are listed in table 4 .

The error for item 1 is estimated from the reproducibility of individual measurements. Part of this fluctuation is caused by slight drifts in the collecting and bucking potentials and possibly from charging of some of the insulators. 
TABLE 4. Possible errors in rhm determinations

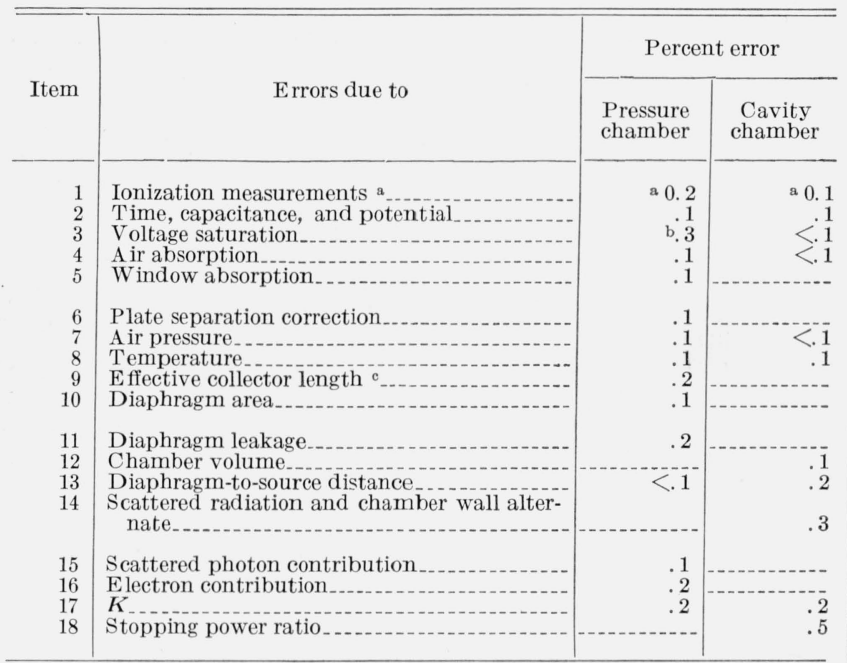

a Of individual points.

b Including relative measurement of currents for different pressures but not correction for error for item 2.

c Including field distortion.

The timing accuracy depends principally on the difference in action time of two relays [12]. An investigation indicates that the accuracy is about 0.01 percent for the times used here. The condenser and potentiometer are each calibrated to an accuracy of 0.05 percent but the capacitance drifted during the experiment by almost 0.1 percent. However, it does not seem probable that the determination of the charge collected on the condenser is in error by more than 0.1 percent (item 2).

The accuracy of the saturation extrapolation depends on the adequacy of the Jaffé-Zanstra theory. Each extrapolation is obtained by a least squares fit so there is no subjective error. The amount of extrapolation at $3.96 \mathrm{~atm}$ for cesium-137 is only about $1 \frac{1}{2}$ percent. However, the constancy of $\left(I_{\infty} e^{P d \mu} / P\right.$ in table 1 for cesium-137 and similar values for cobalt-60 (which agree to within $\pm 0.15 \%$ ) indicates that the error in extrapolation is not likely to be over 0.3 percent (item 3). For the cavity chamber the extrapolation is only 0.1 percent so the uncertainty must be small compared to 0.1 percent.

The air attenuation between plates 4 to 7 and the source varies from about 7 percent to 17 percent for cesium-137 and is about 12 percent for cobalt-60. The distance used in the computation is known to at least 0.1 percent. There appears to be no accurate experimental determination of the absorption coefficient for air although the values for aluminum agree with theory to within a few tenths of a percent for the sources considered here. Therefore, it seems reasonable not to expect an error of more than 0.1 percent for item 4 because the maximum correction for air absorption is only about 17 percent for the pressure-chamber measurement. For the cavitychamber measurements, the maximum correction is only about 0.7 percent so the uncertainty in this item should be negligible.
The absorption in the aluminum window of the pressure chamber is of the order of 1 percent. It is difficult to imagine that variations in thickness, absorption coefficient, or composition could cause uncertainties of more than 0.1 percent (item 5).

Several extrapolations for inadequate plate separation were tried. For any reasonable assumption on this extrapolation, the uncertainty is probably not more than 0.1 percent (item 6 ). The small variation with pressure indicated in figures 7 and 8 corroborates this.

The density of air in the pressure chamber is determined from the gage pressure and temperature in the tank and the barometric pressure and room temperature. Errors in the room pressure and temperature could cause less than 0.1 percent error in the air density within the tank at the pressure used. However, as the gage could be calibrated to only $\pm 0.1 \mathrm{psi}$ and it was not calibrated at the exact pressures used, there is a possible uncertainty of the pressure of about 0.1 percent at the higher pressures (item 7). The same uncertainty is estimated for measurements below $5 \mathrm{~atm}$ where the mercury manometer is used. Temperature fluctuation in the tank as indicated by the thermocouples could not have caused an uncertainty in the density determination of more than 0.1 percent (item 8).

For the cavity-chamber measurements, the air pressure is given by a barometer calibrated to 0.1 $\mathrm{mm}$ and the pressure was very constant during a set of measurements. Thus, the error in item 7 should be less than 0.1 percent. However, the temperature did vary by about \pm 0.1 percent during a set of measurements so a possible error of 0.1 percent is assumed for item 8 .

The effective length of the collector plate is determined by the measured length and by any field distortion caused by the presence of the grounded tank and by warpage of the plates. The measured length could not be in error by as much as 0.1 percent but, as indicated earlier, the distortion produced by the grounded tank is determined by ionization means. Therefore, the reproducibility of the ionization measurements $(0.1 \%$ for this investigation $)$ determines this possible error. From previous work [11] it appears that the warpage of the plate could produce an uncertainty of no more than 0.1 percent. Therefore, for these two possible errors it is estimated that the effective plate length could not be in error by more than 0.2 percent (item 9 ).

The diaphragm area is determined by measuring four radii at different depths. These measurements show the hole to be slightly coned (about 3 parts in 7,800 ) at the two ends, but at any one depth the radii agree to 8 parts in 78,000 . The uncertainty in the physical determination of the hole area is therefore not more than 0.1 percent (item 10).

The effective area of the diaphragm is slightly larger than the hole area because of leakage of radiation through the edge of the holes [3]. The increased area amounts to about 1.8 percent for cesium-137 and about 3.5 percent for cobalt-60 radiation. It is estimated that the uncertainty 
[3] in this leakage could not cause an error of more than 0.2 percent (item 11).

The uncertainty in the cavity chamber volume (item 12) is assumed to be the same as that estimated by Attix and Ritz [5] (0.1\%).

The distance between the collimator and the source $(87.1 \mathrm{~cm})$ is determined by a cathetometer and vernier calipers reading to $0.1 \mathrm{~cm}$. It is estimated that the error introduced by the uncertainty of this distance is less than 0.1 percent (item 13). However, the uncertainty of the source-to-cavitychamber distance is somewhat larger -about 0.2 percent.

The accuracy of the correction for scattering from the walls of the room and from the cavity-chamber walls, and for the photon attenuation in the wall is estimated to be the same as that given by Attix and Ritz [5] (item 14).

The computed scattered photon contribution to the ionization for cesium-137 gamma rays amounts to about 4 percent of the electron contribution at $10.85 \mathrm{~atm}$ and about 2 percent at $3.96 \mathrm{~atm}$; for cobalt-60 this contribution amounts to about $3 \frac{1}{2}$ percent for the pressures used. The agreement (see fig. 8) in the scattered photon contribution obtained at various pressures for a given radiation indicates that it is reproducible to about 0.1 percent (item 15).

The agreement (see fig. 7) in the electron contribution obtained at different pressures indicates that this quantity is reproducible to about 0.2 percent (item 16).

The theoretical values of $K$ determined for cobalt60 gamma rays by Roesch [16] are about 0.3 percent larger than those determined by Aglintser and Ostromukhova [15]. Values obtained by Aglintsev and Ostromukhova are used here, but the uncertainty of this correction (item 17) has been assigned a value of 0.2 percent.

Probably the largest single uncertainty in the output determination by the cavity-chamber is in the stopping power ratio (item 18). Three different values for carbon-walled air ionization chambers have been suggested recently. The maximum difference between them is about 2 percent. However, the values labeled "Caldwell" in table 3 do not fit the data of Attix and Ritz [5] and of Whyte, who conducted experiments with cavity chambers having walls of different atomic number. The experimental data from these authors fit the "Bakker and Segre" and "NCRP" stopping-power ratios slightly better than those of "Caldwell" but neither obtained a direct measurement of the stopping power for air. The data of table 3 also indicate better agreement between the free-air chamber and cavity chamber for the "Bakker and Segre" and "NCRP" stoppingpower ratios with the cobalt-60 source but poorer with the cesium-137 source. Attix and Ritz estimate the accuracy of the stopping-power ratios as 0.3 percent, but Whyte gives a value of 0.5 percent. The latter has been adopted for the present report.

The maxim um possible error is obtained by adding the errors of item 2 through 18 , and is 1.9 percent for the pressure chamber and 1.5 percent for the cavity measurements. Item 1 is greatly reduced by the multiplicity of observations. If one assumes that all of these items give random errors, the square root of the sum of their squares gives 0.6 percent for the pressure-chamber method and about 0.7 percent for the cavity-chamber method.

\section{Summary}

1. By a proper choice of the constant in the Jaffé-Zanstra method of extrapolating the ionization current to an infinite field strength, it has been shown that consistent saturation currents can be computed from ionization measurements made with noninfinite fields, at least for pressures of 4 to 12 atm in air. However, the best constant seems to be somewhat different than that previously used. It has also been shown that field strengths per atmosphere of greater than 85 and probably greater than $114 \mathrm{vcm}^{-1} \mathrm{~atm}^{-1}$ are needed for the KaraMichaelova and Lea extrapolation technique.

2. Design data for the construction of free-air chambers used in the measurement of cesium-137 and cobalt-60 gamma rays in roentgens are presented.

3. Determination of the gamma-ray output from a cesium-137 and a cobalt-60 source by means of a free-air chamber and a cavity chamber are indicated. The experiments are not sufficiently accurate to unambiguously decide which value of stoppingpower ratios should be used.

4. It would seem from the estimate of errors that the uncertainty in the two methods of measurement is approximately the same and, therefore, that neither can be designated as the standard method.

The author thanks T. P. Loftus, who made all of the cavity chamber measurements; F. H. Attix, with whom many of the problems arising during the investigation were discussed; and G. H. Dempsey, who was of great assistance in setting up the equipment.

\section{References}

[1] International commission on radiological units and measurements, 1956 Rept., NBS Handbook 62, (1957).

[2] G. W. C. Kaye and W. Binks, The ionization measurements of $\gamma$-radiation, Proc. Roy. Soc. (London)A161, 564 (1937).

[3] L. S. Taylor et al., Measurements in roentgens of the gamma radiation from radium by the free-air ionization chamber, Am. J. Roentgenol. Radium Therapy Nuclear Med. 44, 428 (1940); also J. Research NBS 24, $247(1940)$.

[4] G. R. Grove, Measurement of cesium-137 and cobalt-60 gamma radiation with a pressure ionization chamber, J. Research NBS 48, 147 (1952).

[5] F. H. Attix and V. H. Ritz, A determination of the gamma-ray emission of radium, J. Research NBS $\mathbf{5 9}$, 293 (1957).

[6] W. Miller and R. J. Kennedy, Measurement of field distortion in free-air ionization chambers by an analog method, J. Research NBS 55, 291 (1955).

[7] H. Zanstra, Ein kurzes verfahren zur bestimmung des sättigungsstromes nach der Jafféschen theorie der kolonnenionistron, Physica 2, 817 (1935). 
[8] L. S. Taylor, G. Singer, and A. L. Charlton, Measurement of super voltage X-rays with the free-air ionization chamber, J. Research NBS 21, 19 (1938).

[9] J. W. Boag, Ionization chambers, Radiation Dosimetry (Academic Press, New York, N.Y., 1956).

[10] E. Kara-Michaelova and D. E. Lea, The interpretation of ionization measurements in gases at high pressures, Proc. Cambridge Phil. Soc. 36, 101 (1940).

[11] H. O. Wyckoff and F. H. Attix, Design criteria for freeair ionization chambers, NBS Handbook 64 (1957).

[12] L. Costrell and F. H. Attix, Automatic timer simplifies small-current measurement, Nucleonics 15, No. 2, 83 (1957).

[13] H. O. Wyckoff and F. S. Kirn, Standard ionizationchamber requirement for 250 to 500 kilovolt X-rays, J. Research NBS 58, 111 (1957).

[14] F. H. Attix and L. DeLaVergne, Plate separation requirements for standard free-air ionization chambers, J. Research NBS 53, 393 (1954).
[15] K. K. Aglintsev and G. P. Ostromukhova, Ionization distribution along the gamma-ray beam and reproduction of the roentgen by means of a standard ionization chamber, Atomnaya energ. 6, 63 (1959).

[16] W. C. Roesch, Dose for nonelectronic equilibrium conditions, Radiation Research 9, 399 (1958).

[17] G. White Grodstein, X-ray attenuation coefficients from $10 \mathrm{kev}$ to $100 \mathrm{Mev}$, NBS Circ. 583 (1957).

[18] W. C. Roesch (private communication).

[19] G. Minton (private communication)

[20] G. N. Whyte, Measurement of the Bragg-Gray stopping power correction, Radiation Research 6, 371 (1957).

Washington, D.C.

Paper (64C2-28) 